\title{
Statement for gastroesophageal reflux disease after peroral endoscopic myotomy from an international multicenter experience
}

\author{
Haruhiro Inoue ${ }^{1}$ Hironari Shiwaku ${ }^{2}$ - Yasutoshi Kobayashi ${ }^{3}$. Philip W. Y. Chiu ${ }^{4} \cdot$ Robert H. Hawes $^{5}$. \\ Horst Neuhaus $^{6} \cdot$ Guido Costamagna $^{7} \cdot$ Stavros N. Stavropoulos $^{8} \cdot$ Norio Fukami $^{9} \cdot$ Stefan Seewald $^{10}$. \\ Manabu Onimaru ${ }^{1} \cdot$ Hitomi Minami $^{11}$. Shinwa Tanaka ${ }^{12}$. Yuto Shimamura ${ }^{1}$. Esperanza Grace Santi ${ }^{13}$. \\ Kevin Grimes ${ }^{14} \cdot$ Hisao Tajiri ${ }^{15}$
}

Received: 26 March 2019 / Accepted: 28 August 2019 / Published online: 26 September 2019

(c) The Author(s) 2019

\begin{abstract}
It has been 10 years since peroral endoscopic myotomy (POEM) was reported for the first time, and POEM has currently become the standard treatment for achalasia and related disorders globally because it is less invasive and has a higher curative effect than conventional therapeutic methods. However, there are limited studies comparing the long-term outcomes of POEM with those of conventional therapeutic methods, particularly in the occurrence of gastroesophageal reflux disease (GERD) after therapy. With this background, we held a consensus meeting to discuss the pathophysiology and management of GERD after POEM based on published papers and experiences of each expert and to discuss the prevention of GERD and dealing with anti-acid drug refractory GERD. This meeting was held on April 27, 2018 in Tokyo to establish statements and finalize the recommendations using the modified Delphi method. This manuscript presents eight statements regarding GERD after POEM.
\end{abstract}

Keywords Achalasia $\cdot$ GERD $\cdot$ Myotomy

Haruhiro Inoue

haruinoue777@yahoo.co.jp

Hironari Shiwaku

hiro.shiwaku@gmail.com

1 Digestive Diseases Center, Showa University Koto-Toyosu Hospital, Toyosu 5-1-38, Koto-Ku, Tokyo 135-8577, Japan

2 Department of Gastroenterological Surgery, Fukuoka University Faculty of Medicine, 7-45-1, Nanakuma, Jyonan-ku, Fukuoka 814-0180, Japan

3 Department of Gastroenterology and Hepatology, Jichi Medical University, Shimotsuke, Tochigi, Japan

4 The Institute of Digestive Disease, Faculty of Medicine of the Chinese University of Hong Kong, Hong Kong, China

5 Center for Interventional Endoscopy, Florida Hospital Orlando, Orlando, Florida, USA

6 Department of Internal Medicine, Evangelisches Krankenhaus Düsseldorf, Düsseldorf, Germany

7 Digestive Endoscopy Unit, Fondazione Policlinico Universitario A. Gemelli IRCCS, Catholic University, Rome, Italy
8 Division of Gastroenterology, Hepatology, and Nutrition, NYU-Winthrop Hospital, New York, USA

9 Division of Gastroenterology and Hepatology, Mayo Clinic Arizona, Scottsdale, AZ, USA

10 Centre of Gastroenterology, Klinik Hirslanden, Zurich, Switzerland

11 Department of Gastroenterology and Hepatology, Nagasaki University, Nagasaki, Japan

12 Division of Gastroenterology, Department of Internal Medicine, Graduate School of Medicine, Kobe University, Kobe, Japan

13 Section of Gastroenterology and Digestive Endoscopy, De La Salle University Medical Center, Dasmarinas City, Philippines

14 Department of Surgery, University of Cincinnati College of Medicine, Cincinnati, OH, USA

15 Department of Innovative Interventional Endoscopy Research, The Jikei University School of Medicine, Tokyo, Japan 


\section{Introduction}

It has been 10 years since peroral endoscopic myotomy (POEM) was reported for the first time; currently, POEM has become the standard treatment for achalasia and related disorders worldwide as it is less invasive and has a higher curative effect than conventional therapeutic methods $[1,2]$.

However, there are limited studies comparing the longterm outcomes of POEM with those of conventional therapeutic methods, particularly with respect to the occurrence of gastroesophageal reflux disease (GERD) after therapy [3].

During the ordinary procedure of Heller myotomy (laparotomy or laparoscopic), adjacent structures surrounding the distal esophagus responsible for natural antireflux mechanisms, including the phrenoesophageal ligament, are inevitably circumferentially dissected to perform myotomy on the esophagus. This procedure results in an impairment of the natural antireflux mechanisms and causes postoperative GERD. Therefore, Heller myotomy requires fundoplication to prevent GERD. Several studies showed that POEM achieved similar curative effect compared with Heller myotomy for achalasia [3-6]. However, a fundoplication is not performed during POEM. Therefore, when POEM was first introduced, the development of GERD was a huge concern. However, to date, there have been few reports on the occurrence of GERD requiring surgical intervention with fundoplication [7-10].

With this background, we held a consensus meeting to discuss the pathophysiology and management of GERD after POEM based on published papers and personal experiences from each expert, and to discuss how to prevent GERD and how to deal with GERD refractory to acid suppressing medications when it is encountered. This consensus meeting resulted in eight statements regarding GERD after POEM. This meeting was held on Friday, April 27, 2018, prior to the 3rd Tokyo International Endoscopy Live Course (Tokyo Live 2018).

\section{GERD after POEM-consensus statements development process-consensus statements committee members}

The consensus statements committee comprised sixteen international gastrointestinal endoscopists, including five members of the development committee and fifteen members of the evaluation process as shown in Table 1. The development committee selected the following eight topics as targets for core statements:

(i) The incidence of GERD after POEM (ii) The incidence of late complications of GERD after POEM

(iii) The rate of GERD after POEM and after Heller with partial fundoplication

(iv) The role of anti-acid drugs after POEM

(v) The rate of additional fundoplication for refractory GERD after POEM

(vi) The reasons for the occurrence of GERD after POEM

(vii) The usefulness of the double-scope transillumination technique during POEM as it relates to GERD minimization

(viii) Management for anti-acid drug refractory GERD after POEM.

\section{Evaluation process}

The consensus statement development committee conducted a systematic review on the clinical questions (CQs) further mentioned in the text. Committee members independently searched literature pertinent to the CQs using Medline, Cochrane Library, and Japan Medical Abstract Society Database, starting from 2010 when the first case of POEM for humans was reported up to April 2018 and performed metaanalysis if applicable. After reviewing the final results of the systematic review and meta-analysis for the CQs, development committee members finalized the proposed guideline statements for each CQ assessing the quality of evidence and assigning a strength of recommendation in accordance with the Grading of Recommendations Assessment, Development, and Evaluation (GRADE) tool (Table 2) [11, 12]. The evaluation committee conducted the consensus meeting for GERD after POEM at Tokyo Live 2018 Satellite Symposium. Modified Delphi method was used to reach the consensus by the 15 expert panels. Delphi voting involves a rating scale (1-3, disagree; $4-6$, unsure; $7-9$, agree) and the results are expressed as the median, highest, and lowest [13]. At least $80 \%$ agreement was required for consensus to be reached. Where consensus could not be achieved, statements were revised and another Delphi round was performed. When these processes were complete, the committee released the final version of the manuscript.

Regarding statements with evidence level D, they are written not as CQs but as future research questions (FRQs). The summary of CQ, FRQ and each statement is shown in Table 3 .

\section{Target patients}

These consensus statements apply to patients who are considering POEM or underwent POEM. These consensus statements are intended to be used by the clinicians who 
Table 1 Committee members

\begin{tabular}{|c|c|c|}
\hline \multicolumn{3}{|c|}{ Committee members for the development of statement } \\
\hline 1 & Haruhiro Inoue & Digestive Diseases Center, Showa University Koto-Toyosu Hospital, Tokyo, Japan \\
\hline 2 & Hironari Shiwaku & Department of Gastroenterological Surgery, Fukuoka University, Fukuoka, Japan \\
\hline 3 & Yasutoshi Kobayashi & Department of Gastroenterology and Hepatology, Jichi Medical University, Tochigi, Japan \\
\hline 4 & Manabu Onimaru & Digestive Diseases Center, Showa University Koto-Toyosu Hospital, Tokyo, Japan \\
\hline 5 & Hitomi Minami & Department of Gastroenterology and Hepatology, Nagasaki University, Nagasaki, Japan \\
\hline \multicolumn{3}{|c|}{ Committee members of the evaluation process } \\
\hline 1 & Haruhiro Inoue & Digestive Diseases Center, Showa University Koto-Toyosu Hospital, Tokyo, Japan \\
\hline 2 & Robert H. Hawes & Center for Interventional Endoscopy, Florida Hospital Orlando, Florida, USA \\
\hline 3 & Horst Neuhaus & Department of Internal Medicine, Evangelisches Krankenhaus Düsseldorf, Düsseldorf, Germany \\
\hline 4 & Guido Costamagna & $\begin{array}{l}\text { Digestive Endoscopy Unit, Fondazione Policlinico Universitario A.Gemelli IRCCS, Catholic } \\
\text { University, Rome, Italy }\end{array}$ \\
\hline 5 & Stavros N. Stavropoulos & $\begin{array}{l}\text { Division of Gastroenterology, Hepatology, and Nutrition, NYU-Winthrop Hospital, New York, } \\
\text { USA }\end{array}$ \\
\hline 6 & Philip W.Y. Chiu & $\begin{array}{l}\text { The Institute of Digestive Disease, Faculty of Medicine of the Chinese University of Hong Kong, } \\
\text { Hong Kong, China }\end{array}$ \\
\hline 7 & Norio Fukami & Division of Gastroenterology and Hepatology, Mayo Clinic Arizona,Scottsdale, Arizona, USA \\
\hline 8 & Stefan Seewald & Centre of Gastroenterology, Klinik Hirslanden, Zürich, Switzerland \\
\hline 9 & Hironari Shiwaku & Department of Gastroenterological Surgery, Fukuoka University, Fukuoka, Japan \\
\hline 10 & Manabu Onimaru & Digestive Diseases Center, Showa University Koto-Toyosu Hospital, Tokyo, Japan \\
\hline 11 & Hitomi Minami & Department of Gastroenterology and Hepatology, Nagasaki University, Nagasaki, Japan \\
\hline 12 & Shinwa Tanaka & $\begin{array}{l}\text { Division of Gastroenterology, Department of Internal Medicine, Graduate School of Medicine, } \\
\text { Kobe University, Kobe, Japan }\end{array}$ \\
\hline 13 & Esperanza Grace Santi & $\begin{array}{l}\text { Section of Gastroenterology and Digestive Endoscopy, De La Salle University Medical Center, } \\
\text { Dasmarinas City, Philippines }\end{array}$ \\
\hline 14 & Kevin Grimes & Department of Surgery, University of Cincinnati College of Medicine, Cincinnati, OH, USA \\
\hline 15 & Hisao Tajiri & $\begin{array}{l}\text { Department of Innovative Interventional Endoscopy Research, The Jikei University School of } \\
\text { Medicine, Tokyo, Japan }\end{array}$ \\
\hline
\end{tabular}

Table 2 Evidence level and strength of recommendation

Grades of recommendation
1: Strong recommendation
2: Weak recommendation
N/A: Unclear recommendation, or recommendation grade cannot be
determined
Evidence level
A: Based on strong evidence
B: Based on moderate evidence
C: Based on weak evidence
D: Based on very weak evidence

engage in POEM practice and other clinicians providing aid for the digestive issues of patients undergoing POEM. These statements provide general recommendations regarding current standards of care for POEM procedures and, therefore, each statement's users should carefully recognize that its application in clinical practice may need to be individualized according to each clinician's background and resources, and patients' background, preference, age, comorbidity, and social and medical conditions.
The consensus statements committee comprised sixteen international gastrointestinal endoscopists, including five members of the development committee and fifteen members of the evaluation process.

Recommendations can be categorized as strong, weak, or unclear.

Recommendations involve a trade-off between benefits and harms. Those making a recommendation should consider four main factors:

1. The trade-offs, considering the estimated size of the effect for the main outcomes, the confidence limits around those estimates, and the relative value placed on each outcome.

2. The quality of evidence

3. Translation of the evidence into practice in a specific setting, considering important factors that could modify the size of the expected effects, such as proximity to a hospital or availability of necessary expertise.

4. Uncertainty about baseline risk for the population of interest. If there is uncertainty about translating the evidence into practice in a specific setting, or uncertainty 
Table 3 Summary of statement

\begin{tabular}{|c|c|c|c|c|}
\hline & CQ and FRQ & Statement & $\begin{array}{l}\text { Strength of } \\
\text { recommenda- } \\
\text { tion }\end{array}$ & $\begin{array}{l}\text { Evi- } \\
\text { dence } \\
\text { Level }\end{array}$ \\
\hline CQ1 & What is the incidence of GERD after POEM? & $\begin{array}{l}\text { POEM may induce GERD, but incidence depends on } \\
\text { measurement }\end{array}$ & N/A & $\mathrm{B}$ \\
\hline CQ2 & $\begin{array}{l}\text { Are there any reports of stenosis, bleeding, or Barrett's } \\
\text { esophageal cancer due to GERD after POEM? }\end{array}$ & $\begin{array}{l}\text { The incidence of late complications of GERD after } \\
\text { POEM seems to be low; however, further long-term } \\
\text { investigation is needed }\end{array}$ & N/A & $\mathrm{C}$ \\
\hline CQ3 & $\begin{array}{l}\text { Is post-POEM GERD higher than GERD after laparo- } \\
\text { scopic Heller-Dor? }\end{array}$ & $\begin{array}{l}\text { Based on current data, GERD after POEM occurs } \\
\text { more frequently than after Heller plus partial fun- } \\
\text { doplication }\end{array}$ & N/A & $\mathrm{C}$ \\
\hline FRQ 4 & $\begin{array}{l}\text { What is the role of proton pump inhibitor (PPI) after } \\
\text { POEM? }\end{array}$ & $\begin{array}{l}\text { Most patients with post-POEM GERD respond to PPI } \\
\text { therapy; however, the indications for PPI are not } \\
\text { well defined }\end{array}$ & N/A & $\mathrm{D}$ \\
\hline FRQ 5 & $\begin{array}{l}\text { What is the rate of cases where additional fundopli- } \\
\text { cation was performed for refractory GERD after } \\
\text { POEM? }\end{array}$ & $\begin{array}{l}\text { The need for fundoplication to treat GERD after } \\
\text { POEM is extremely low }\end{array}$ & N/A & $\mathrm{D}$ \\
\hline FRQ 6 & $\begin{array}{l}\text { Why is the rate of GERD high in POEM which } \\
\text { preserves the periesophageal suspensory ligaments } \\
\text { involved in natural antireflux mechanisms? }\end{array}$ & $\begin{array}{l}\text { Excessive gastric myotomy and incision of the collar } \\
\text { sling fibers may increase the frequency of GERD } \\
\text { after POEM }\end{array}$ & N/A & $\mathrm{D}$ \\
\hline FRQ 7 & $\begin{array}{l}\text { Is the double-scope transillumination method helpful } \\
\text { for controlling the length of myotomy? }\end{array}$ & $\begin{array}{l}\text { Currently, the double-scope method is the most } \\
\text { reliable way to confirm the length and direction of } \\
\text { myotomy on the gastric side }\end{array}$ & 1 & $\mathrm{D}$ \\
\hline FRQ 8 & $\begin{array}{l}\text { How should the patient with medication refractory } \\
\text { GERD after POEM be managed? }\end{array}$ & $\begin{array}{l}\text { For refractory severe post-POEM GERD, some antire- } \\
\text { flux procedure may be considered }\end{array}$ & 2 & $\mathrm{D}$ \\
\hline
\end{tabular}

about baseline risk, this may lower our confidence in a recommendation.

Strong evidence: further research is unlikely to change our confidence in the estimate of effect.

Moderate evidence: further research is likely to have an important impact on our confidence in the estimate of effect and may change the estimate

Weak evidence: further research is extremely likely to have an important impact on our confidence in the estimate of effect and may change the estimate

Very weak evidence: any estimate of effect is extremely uncertain.

CQ1: what is the incidence of GERD after POEM?

Statement: POEM may induce GERD, but incidence depends on measurement.

Strength of recommendation: N/A

Evidence level: B

Delphi scores: Median $=8$, Lowest $=7$, Highest $=9$

Commentary: according to several meta-analyses, symptomatic GERD after POEM occurred in $8.5-19 \%$ of patients [3, 14, 15]. The endoscopic findings of erosive esophagitis after POEM were detected in 13-29.4\% of patients, whereas abnormal $24 \mathrm{pH}$ study results were observed in $39-47.5 \%$ of patients $[3,14,15]$. In general, the rate of GERD after POEM depends on the type of measurement and there is significant difference between symptoms, endoscopic evidence, and $\mathrm{pH}$ measurement. In the future, a unified definition of GERD after POEM should be established to standardize the assessment of the incidence of GERD after POEM.

CQ2: are there any reports of stenosis, bleeding, or Barrett's esophageal cancer due to GERD after POEM?

Statement: the incidence of late complications of GERD after POEM seems to be low; however, further long-term investigation is needed.

Delphi scores: Median $=8.5$, Lowest $=7$, Highest $=9$

Strength of recommendation: N/A

Evidence level: $\mathrm{C}$

Commentary: currently, there is no published report on the development of GERD with refractory stenosis, bleeding, or Barrett's esophagus and carcinoma after POEM. However, there are verbal reports on a few cases of stenosis and bleeding due to GERD after POEM from high volume centers. In addition, although there were institutions that experienced the occurrence of SSBE (short segment Barrett Esophagus) associated with erosive esophagitis after POEM, there was no report on the occurrence of LSBE (long segment Barrett Esophagus). To date, Barrett's related esophageal cancer after POEM has not been reported in the literature or by any of the expert centers. The panel recommended long-term follow-up and report of the clinical outcomes for patients with GERD after POEM to monitor GERD-related complications. 
CQ3: is post-POEM GERD higher than GERD after laparoscopic Heller-Dor?

Statement: based on the current data, GERD after POEM occurs more frequently than after Heller plus partial fundoplication.

Strength of recommendation: N/A

Evidence level: C

Delphi scores: Median $=8$, Lowest $=6$, Highest $=9$

Commentary: according to the meta-analyses by Schlottmann et al. and Repici et al., gastroesophageal reflux after POEM measured by $\mathrm{pH}$ monitoring was significantly higher than that of Heller-Dor operation [3, 14]. Other reports demonstrated no significant difference in $\mathrm{pH}$ monitoring between POEM and Heller myotomy [16].

The results from the studies which focused on endoscopic evidence of erosive esophagitis and symptomatic GERD comparing POEM and Heller myotomy are variable. Hence, there are no definitive conclusions [17, 18].

FRQ 4: what is the role of proton pump inhibitor (PPI) after POEM?

Statement: most patients with post-POEM GERD respond to PPI therapy; however, the indications for PPI are not well defined.

Strength of recommendation: N/A

Evidence level: D

Delphi scores: Median $=8$, Lowest $=7$, Highest $=9$

Commentary: PPI is effective for the treatment of GERD after POEM [15]. According to a meta-analysis reported by Repici, the rate of PPI use ranged from 2.6 to $27.8 \%$ after POEM [14]. Most patients with post-POEM GERD respond to PPI therapy; however, the indication for use of PPIs after POEM is not well defined. Numerous studies reporting on the occurrence of GERD after POEM demonstrated that there is a discrepancy among the clinical symptoms of GERD, objective assessment with $24 \mathrm{~h} \mathrm{pH}$ study, and endoscopic evidence of esophagitis $[3,14,15]$. Contrary to our general understanding that the prevalence of GERD symptoms in the general population is commonly higher than the rate of positive $\mathrm{pH}$ studies or the rate of endoscopic evidence of esophagitis, in achalasia patients receiving POEM, the $\mathrm{pH}$ study positivity and endoscopic esophagitis rates are usually higher than symptomatic reflux. This results in uncertainties regarding the use of PPIs after POEM, whether it is for symptomatic relief or healing esophagitis.

FRQ 5: what is the rate of cases where additional fundoplication was performed for refractory GERD after POEM?

Statement: the need for fundoplication to treat GERD after POEM is extremely low.

Strength of recommendation: N/A

Evidence level: D

Delphi scores: Median $=8$, Lowest $=7$, Highest $=9$
Commentary: currently, there are a few reports which describe additional fundoplication for GERD after POEM [7-9]. Swanström states that the need for an antireflux procedure after POEM is exceedingly rare in his and other Western experiences [10]. According to multicenter retrospective study in Japan (Over 2000 cases), the rate of cases in which additional surgical fundoplication was performed for GERD after POEM was $0.1 \%$ (2 cases) (Unpublished data. Reported in this consensus meeting) [19].

FRQ 6: why is the rate of GERD high in POEM which preserves the periesophageal suspensory ligaments involved in natural antireflux mechanisms?

Statement: excessive gastric myotomy and incision of the collar sling fibers may increase the frequency of GERD after POEM.

Strength of recommendation: N/A

Evidence level: D

Delphi scores: Median $=8$, Lowest $=6$, Highest $=9$

Commentary: in patients with achalasia, esophageal peristalsis affecting acid clearance is impaired. Therefore, even when performing Heller myotomy or performing POEM, postoperative GERD after incision of LES should be considered. Simic et al. reported that postoperative GERD occurs in $23.1 \%$ patients after Heller myotomy with full mobilization of abdominal esophagus around the hiatus [20]. Alternatively, preservation of the phrenoesophageal ligament during Heller myotomy can reduce the development of GERD, regardless of whether fundoplication was performed (GERD after Heller with limited hiatal dissection + Dor: $8.5 \%$; GERD after Heller with limited hiatal dissection alone without fundopication: 9.1\%). According to this theory, POEM which naturally preserves the phrenoesophageal ligament should reduce the development of GERD. However, the clinical rate of GERD after POEM remains high. Factors other than preservation of the phrenoesophageal ligament may have contributed to the occurrence of GERD after POEM [3, $14,15]$. According to the results of a multicenter retrospective study in Japan (Over 2000 cases, nonpublished data), it was suggested that excessive muscle incision of longer than $4 \mathrm{~cm}$ in the gastric cardia on the posterior side may be responsible for erosive esophagitis over Grade C (Unpublished data. Reported in this consensus meeting) [19]. Moreover, according to a single-center report by Fukuoka University, incision of the sling fibers (oblique muscle) at the gastric cardia may be the cause of significant GERD, leading to erosive esophagitis above Grade C (Unpublished data. Reported in this consensus meeting) [19]. From these results, the direction of myotomy is recommended anterior (1-2 o'clock) or posterior (4-5 o'clock) side which could preserve oblique muscle and the length of myotomy in gastric side is recommended $2-3 \mathrm{~cm}$. Future studies should address the extent of myotomy, particularly to the gastric 
cardia, as well as the effect of anatomical dissection of the sling fibers on the occurrence of GERD after POEM.

FRQ 7: is the double-scope transillumination method helpful for controlling the length of myotomy?

Statement: currently, the double-scope method is the most reliable way to confirm the length and direction of myotomy on the gastric side.

Strength of recommendation: 1

Evidence level: D

Delphi scores: Median $=8$, Lowest $=6$, Highest $=9$

Commentary: originally, the double-scope method was proposed as a means to prevent incomplete myotomy of lower esophageal sphincter (LES) during POEM (Figs. 1 and 2) $[21,22]$. The double-scope technique comprised observing the extent of submucosal tunnel development at the gastric cardia using an ultrathin endoscope while the original endoscope transilluminates through the mucosa while being at the end of the tunnel. Clinical outcomes from a multicenter retrospective study in Japan showed that excessive muscle incision of longer than $4 \mathrm{~cm}$ in the gastric cardia on the posterior side may result in the development of erosive esophagitis above LA Grade C after POEM (Unpublished data. Reported in this consensus meeting) [19]. Furthermore, according to the experience reported by Fukuoka University,

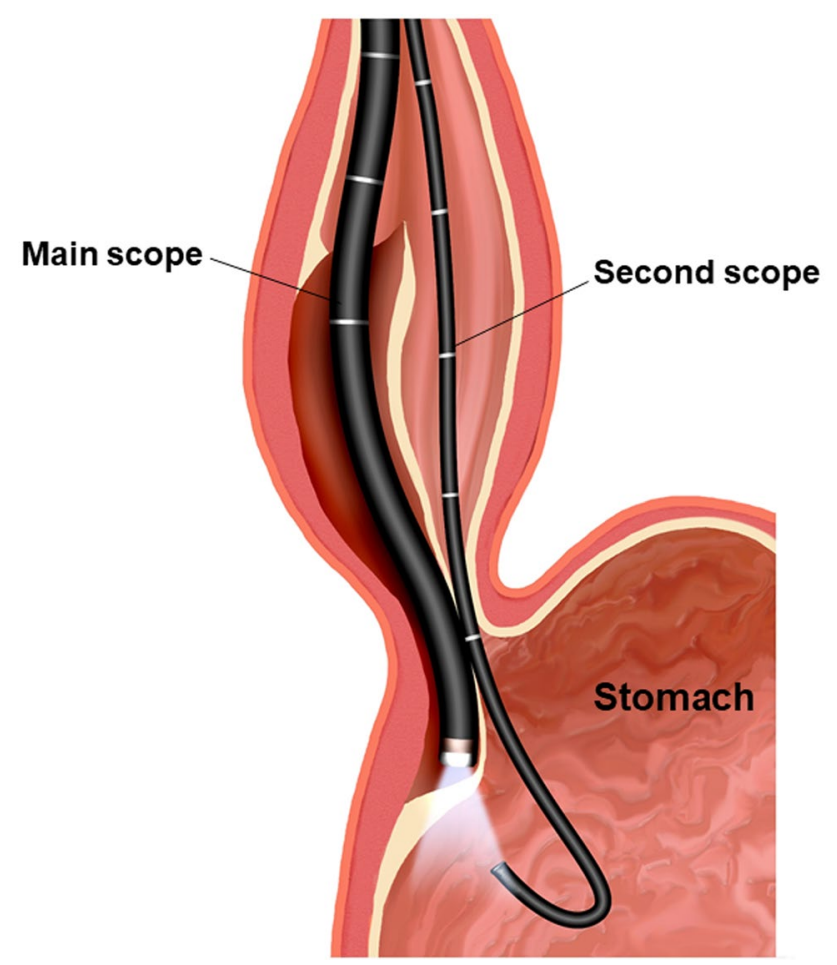

Fig. 1 The image of double-scope method. A second endoscope was inserted into the stomach to examine the cardia region. If the procedure reached the gastric side, the light from the main scope within the submucosal space was visible through the second scope in the stomach

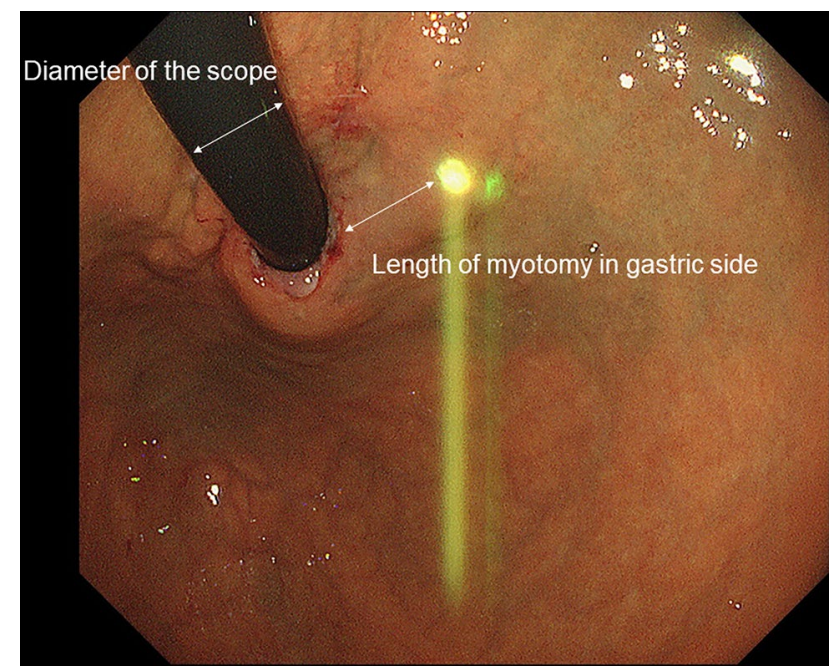

Fig. 2 Endoscopic finding of double scope. The length of myotomy in gastric side can be measured based on the diameter of second scope

preserving the oblique muscle may reduce the occurrence of GERD after POEM (Unpublished data. Reported in this consensus meeting) [19]. The double-scope technique is the most reliable method to confirm the length and direction of myotomy when observing the gastric cardia side. Therefore, it is recommended that operators should perform the double-scope method during POEM to confirm the extent of myotomy in the gastric cardia and reduce GERD after POEM.

CQ8: how should the patient with medication refractory GERD after POEM be managed?

Statement: for refractory severe post-POEM GERD, some antireflux procedures may be considered.

Strength of recommendation: 2

Evidence level: D

Delphi scores: Median $=7$, Lowest $=5$, Highest $=9$

Commentary: most studies report the use of anti-acid therapy as first-line treatment for post-POEM GERD. Most cases respond to PPI [15]. Antireflux surgery will be considered for patients who have significant GERD symptoms and when PPI cannot completely relieve these symptoms. Antireflux surgery achieves control of gastroesophageal reflux through correction of local anatomical defects, including the damaged antireflux mechanisms such as the defective lower esophageal sphincter, oblique muscle and phrenoesophageal ligament. Published reports on antireflux surgery for the management of GERD after POEM are limited. POEM $+\mathrm{F}$ (POEM plus endoscopic fundoplication) may be another choice of treatment [23]. Currently, laparoscopic partial fundoplication (Dor or Toupet) is the most common surgical treatment and this is a routine antireflux procedure after laparoscopic Heller myotomy. According to a multicenter 
retrospective study in Japan (Over 2000 cases), the number of patients with severe GERD that required surgical fundoplication after POEM was $0.1 \%$ ( 2 cases) (Unpublished data. Reported in this consensus meeting) [19]. Although surgical fundoplication is an effective treatment for GERD, it is less than ideal to perform laparoscopic partial fundoplication after a successful endoscopic myotomy. In the future, research should focus on refining the techniques of POEM to minimize occurrence of GERD, as well as developing effective endoscopic antireflux procedures for the management of GERD after POEM.

\section{Conclusion}

According to the results of this consensus meeting and the present published data, it was confirmed that most patients with post-POEM GERD respond to PPI therapy and fundoplication for refractory GERD is rarely needed in the decade-long POEM global experience. PPI remains the mainstay therapy for GERD after POEM with an extremely high rate of efficacy. There are limited data on the use of endoscopic antireflux procedures for the treatment of postPOEM GERD. Intriguing preliminary retrospective data from Japan suggest that POEM technique modifications that focus on limiting the length of myotomy and preserving the collar sling fibers may decrease the frequency of GERD after POEM. It is recommended that a multicenter prospective study evaluating these technique refinements should be considered in the near future.

Acknowledgements This meeting is partially supported by educational grant from Olympus to Tokyo Live 2018.

\section{Compliance with ethical standards}

Ethical Statement This article does not contain any studies with human participants or animals performed by any of authors.

Conflict of interest Dr. Inoue reports grants from Olympus Co., grants from Boston Scientific Co., outside the submitted work; the other authors have nothing to disclose.

Open Access This article is distributed under the terms of the Creative Commons Attribution 4.0 International License (http://creativeco mmons.org/licenses/by/4.0/), which permits unrestricted use, distribution, and reproduction in any medium, provided you give appropriate credit to the original author(s) and the source, provide a link to the Creative Commons license, and indicate if changes were made.

\section{References}

1. Inoue $\mathrm{H}$, Minami $\mathrm{H}$, Kobayashi $\mathrm{Y}$, et al. Peroral endoscopic myotomy (POEM) for esophageal achalasia. Endoscopy. 2010;42:265-71.
2. Inoue $\mathrm{H}$, Shiwaku $\mathrm{H}$, Iwakiri $\mathrm{K}$, et al. Clinical practice guidelines for peroral endoscopic myotomy. Dig Endosc. 2018;30:563-79.

3. Schlottmann F, Luckett DJ, Fine J, et al. Laparoscopic heller myotomy versus peroral endoscopic myotomy (POEM) for achalasia: a systematic review and meta-analysis. Ann Surg. 2018;267:451-60.

4. Talukdar R, Inoue H, Nageshwar Reddy D. Efficacy of peroral endoscopic myotomy (POEM) in the treatment of achalasia: a systematic review and meta-analysis. Surg Endosc. 2015;29:3030-46.

5. Zhang Y, Wang H, Chen X, et al. Per-oral endoscopic myotomy versus laparoscopic heller myotomy for achalasia: a meta-analysis of nonrandomized comparative studies. Medicine (Baltimore). 2016;95:e2736.

6. Patel K, Abbassi-Ghadi N, Markar S, et al. Peroral endoscopic myotomy for the treatment of esophageal achalasia: systematic review and pooled analysis. Dis Esophagus. 2016;29:807-19.

7. Zak Y, Meireles OR, Rattner DW. Laparoscopic Toupet fundoplication for GERD after POEM. 2018 SAGES Meeting/16th World Congress of Endoscopic Surgery. 2018. https://www. sages.org/meetings/annual-meeting/abstracts-archive/lapar oscopic-toupet-fundoplication-for-gerd-after-poem/. Accessed 19 Mar 2019.

8. Kumta NA, Kedia P, Sethi A, et al. Transoral incisionless fundoplication for treatment of refractory GERD after peroral endoscopic myotomy. Gastrointest Endosc. 2015;81:224-5.

9. Tyberg A, Choi A, Gaidhane M, et al. Transoral incisional fundoplication for reflux after peroral endoscopic myotomy: a crucial addition to our arsenal. Endosc Int Open. 2018;6:E549-52.

10. Swanstrom LL. POEM: the sun rises in the east. Gastrointest Endosc. 2018;87:1413-4.

11. Guyatt GH, Oxman AD, Kunz R, et al. What is "quality of evidence" and why is it important to clinicians? BMJ. 2008;336:995-8.

12. Andrews JC, Schunemann HJ, Oxman AD, et al. GRADE guidelines: 15 . Going from evidence to recommendation-determinants of a recommendation's direction and strength. J Clin Epidemiol. 2013;66:726-35.

13. Shiffman RN, Shekelle P, Overhage JM, et al. Standardized reporting of clinical practice guidelines: a proposal from the conference on guideline standardization. Ann Intern Med. 2003;139:493-8.

14. Repici A, Fuccio L, Maselli R, et al. GERD after per-oral endoscopic myotomy as compared with Heller's myotomy with fundoplication: a systematic review with meta-analysis. Gastrointest Endosc. 2018;87(934-43):e18.

15. Akintoye E, Kumar N, Obaitan I, et al. Peroral endoscopic myotomy: a meta-analysis. Endoscopy. 2016;48:1059-68.

16. Bhayani NH, Kurian AA, Dunst CM, et al. A comparative study on comprehensive, objective outcomes of laparoscopic Heller myotomy with per-oral endoscopic myotomy (POEM) for achalasia. Ann Surg. 2014;259:1098-103.

17. Chan SM, Wu JC, Teoh AY, et al. Comparison of early outcomes and quality of life after laparoscopic Heller's cardiomyotomy to peroral endoscopic myotomy for treatment of achalasia. Dig Endosc. 2016;28:27-32.

18. Peng L, Tian S, Du C, et al. Outcome of peroral endoscopic myotomy (POEM) for treating achalasia compared with laparoscopic heller myotomy (LHM). Surg Laparosc Endosc Percutan Tech. 2017;27:60-4.

19. Online presentation; Shiwaku H, Inoue $\mathrm{H}$ et al. Title of presentation: multicenter collaborative retrospective review of peroral endoscopic myotomy for esophageal achalasia. 2018 SAGES Meeting/16th World Congress of Endoscopic Surgery. 2018. https://www.sages.org/video/multicenter-collaborative-retrospect 
ive-review-of-peroral-endoscopic-myotomy/. Accessed 19 March 2019.

20. Simic AP, Radovanovic NS, Skrobic OM, et al. Significance of limited hiatal dissection in surgery for achalasia. J Gastrointest Surg. 2010;14:587-93.

21. Baldaque-Silva F, Marques M, Vilas-Boas F, et al. New transillumination auxiliary technique for peroral endoscopic myotomy. Gastrointest Endosc. 2014;79:544-5.

22. Grimes KL, Inoue H, Onimaru M, et al. Double-scope per oral endoscopic myotomy (POEM): a prospective randomized controlled trial. Surg Endosc. 2016;30:1344-51.
23. Inoue H, Ueno A, Shimamura Y, et al. Peroral endoscopic myotomy and fundoplication: a novel NOTES procedure. Endoscopy. 2019;51(2):161-4.

Publisher's Note Springer Nature remains neutral with regard to jurisdictional claims in published maps and institutional affiliations. 